\title{
Optimization of CC-Coil Design for Wireless Power Transfer System with Series-Series Magnetic Resonance Compensation Technique
}

\author{
Muhammad Muhaimin Mohd Taib ${ }^{1}$, Asmarashid Ponniran ${ }^{1 *}$ \\ ${ }^{1}$ Faculty of Electrical and Electronics Engineering, \\ Universiti Tun Hussein Onn Malaysia, Parit Raja, Batu Pahat, 86400, MALAYSIA
}

DOI: https://doi.org/10.30880/jeva.2021.02.02.006

Received 30 June 2021; Accepted 04 August 2021; Available online 30 December 2021

\begin{abstract}
This study aims to increase the coupling coefficient of the coils and power transfer efficiency (PTE) of the wireless power transfer (WPT) system. WPT system has a severe issue with the PTE as the transfer distance between the transmitter and receiver increases. Therefore, the transmitter and receiver of the single-circular coil (CC-coil) need to be optimized in geometry to maintain high coupling at an optimum distance. Ferrite and aluminum shielding are also crucial on CC-coil optimization. Implementing the series-series (S-S) magnetic resonance compensation technique can increase the PTE of the WPT system. Therefore, the CC-coil is optimized using Ansys Electronics Desktop and co-simulated with the magnetic resonance circuit using Ansys Twin Builder. The results show that the CC-coils' coupling coefficient increased by $21.38 \%$ with the shielding implementation. The maximum optimum transfer distance of $37 \mathrm{~mm}$ for horizontal misalignment and $30 \mathrm{~mm}$ for vertical misalignment. Implementing the S-S magnetic resonance compensation technique can improve the PTE and output power of the WPT system. The power transmitted also varied with the transfer distance, which caused the system's variation of input impedance. Hence, it is essential to consider the coil design and compensation circuit to achieve high PTE and output power at a higher transfer distance.
\end{abstract}

Keywords: Wireless power transfer, series-series magnetic resonance coupling, circular single coil, power transfer efficiency

\section{Introduction}

The development of wireless power transfer (WPT) began in the late 19th century by Nikola Tesla patented the Tesla coil [1]. However, WPT development that used far-field type by Tesla was stunted because of health and safety issues [2]. Microwave wireless power transmission, which is far-field, can cause serious health diseases like cancer [3]. Numerous papers about microwave bioeffects have been published to explain this condition since 1950. Moreover, the latest far-field type technology like laser-based power beaming still does not resolve the health hazard issue, making the application experimental and verification stage. The development of WPT become active back in 2007 when WiTricity from MIT had successfully transmitted $60 \mathrm{~W}$ for 2 meters by using near-field type. This development can solve the charging issue in numerous applications, especially biomedical and electric vehicles (EV) [4]. However, WPT has a severe problem with the power transfer efficiency (PTE) as it decreases when the transfer distance increases. WPT issue related to PTE and transmission coil offset cause the WPT research progress is still theoretical and experimental verification [5], [6].

Generally, the PTE of the WPT is highly dependent on the coupling coefficient between transmitter and receiver. Similar to the transformer concept, the coupling coefficient $(\mathrm{k})$ closed to 1 is tightly coupled with better PTE. The 
coupling coefficient between the transmitter and receiver can be increased by considering the coils' size, shape, and arrangement. However, suppose this parameter is exaggerated increase or decrease. It will introduce other losses that will affect the PTE and not be convenient for specific applications [6]. Therefore, the transmitter and receiver must be optimized in size, shape, and arrangement to achieve a high coupling coefficient at an optimal transfer distance. A circular shape with a single-coil arrangement is chosen in this study, which is called CC-coil. The single transmitter single receiver (STSR) arrangement has less design complexity compared to the other arrangement, which is multiple transmitter single receiver (MTSR) and multiple transmitters multiple receivers (MTMR). Previous studies from [7], [8] claims that circular coil has the best coupling coefficient among other geometries like square and rectangular. One possible factor is that the circular coil does not have any sharp edge cause the coil's eddy current is minimized [9].

WPT system can be further optimized by implementing a magnetic resonance coupling technique. The magnetic resonance coupling technique has two types which are mono-resonant and multi-resonant compensation [10]. Monoresonant compensation comprises four topologies which are series-series (S-S), series-parallel (S-P), parallel-series (P$\mathrm{S}$ ), and parallel-parallel (P-P). Inductor-capacitor-inductor (LCL-type) and inductor-capacitor-capacitor (LCC-type) is a multi-resonant compensation topology. All of these topologies have their advantages and disadvantages. However, the magnetic resonance coupling technique can further increase the power transfer efficiency (PTE) of WPT, even the coupling coefficient between the coils is low. Based on [11], the WPT circuit can have 18 W output power by using magnetic resonance even if the coupling coefficient between the transmitter and receiver is less than 0.1 . Therefore, the magnetic resonance coupling technique is more efficient than other types of WPT as the resonance frequency increases the transfer distance up to mid-range [12].

This study's primary goal is to optimize the parameters of the single circular coil (CC-coil) to achieve a high coupling coefficient at an optimal distance. Besides, series-series magnetic resonance coupling technique is implemented without changing the configuration and arrangement to observe the performance of the WPT, especially in terms of power transfer efficiency (PTE). It is expected that high power received with power transfer efficiency (PTE) can be achieved even the transfer distance increase by considering the proposed method.

\section{WPT System Design}

Wireless power transfer (WPT) has three critical aspects: power electronics circuit, compensation circuit for magnetic resonance with the transmitter, and receiver coil shown in Fig. 1. The frequency from the AC grid (230 V, 50 $\mathrm{Hz})$ needs to be altered to the resonant frequency $(6.78 \mathrm{MHz})$. Then, the transmitter coil converted electrical energy into an electromagnetic wave using magnetic resonance coupling induced by the receiver coil. The receiver coil will convert back the electromagnetic wave into AC electrical energy and rectified into the DC source that followed the load requirement. In this study, the load is a mobile phone charger application that is assumed to be $150 \Omega$ resistor with a maximum output power of $100 \mathrm{~W}$ to simplify the analysis. The transmitter and receiver coil is designed using Ansys Electronics Desktop 2019 R3 under Maxwell 3D component. Then, the compensation circuit and switching circuit is co-simulated with the designed coil using Ansys Twin Builder 2019 R3 under the Simplorer feature.

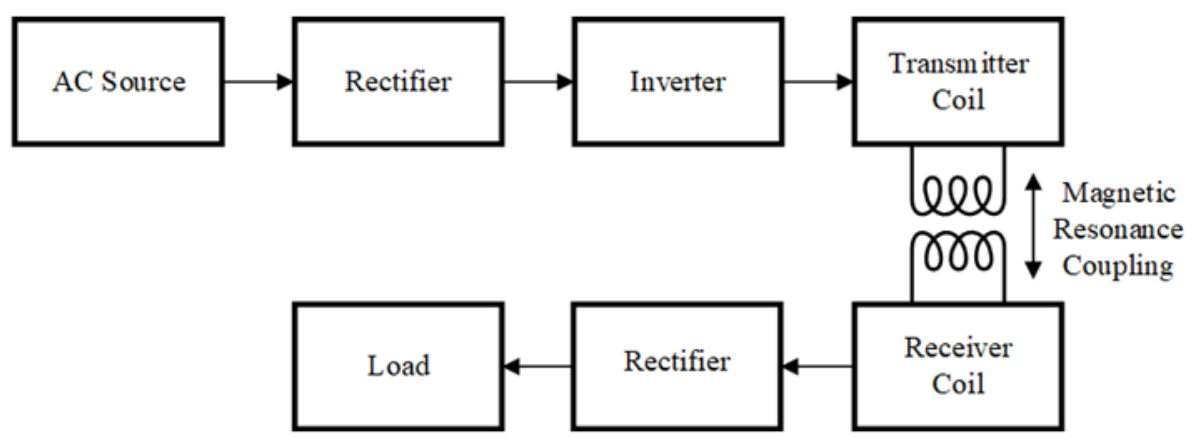

Fig. 1 - Block diagram of WPT

\subsection{Design and Optimization of CC-coils}

In coil design, the determination of mutual inductance of the coils needs to determine as high as possible to increase the coupling coefficient shown in Eq. 1.

$$
k=\frac{M}{\sqrt{L_{T x} L_{R x}}}
$$


However, the determination of mutual inductance using the analytical method required a complex numerical equation. Therefore, the Finite Element Method (FEM) is essential to design, analyze and optimize the coil under Maxwell 3D. Then, the Maxwell 3D model design can be varied in terms of transfer distance, making it valid in a realworld application.

According to [6], several parameters must be considered to design CC-coil, which are the outer diameter (Dout), inner diameter (Din), the width of wire (w), pitch (s), and the number of turns (N) shown in Fig. 2. User-defined primitive under the RectHelix option in Maxwell 3D provide more straightforward ways to draw and design the CCcoil by inserting the value of CC-coil parameters.

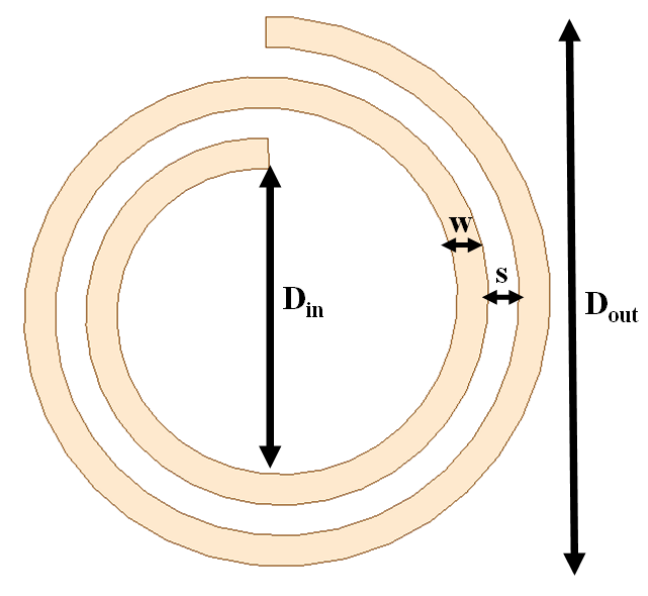

Fig. 2 - Parameter in designing CC-coil

The optimization of CC-coil can be done by increasing the outer diameter, the number of turns, and the width of the wire. On the other hand, the inner diameter of the coil and pitch need to decrease to achieve a high coupling coefficient. Several constrain or requirement has been set to make CC-coil design easier that shown in Table 1 . The constrain value in designing the coil is considered based on the mobile phone application. For example, the maximum length of the coil is set to $100 \mathrm{~mm}$ that mimics most of the mobile phone sizes in the market.

Table 1 - Constrain in designing the coil

\begin{tabular}{cc}
\hline Constrain & Parameter \\
\hline Minimum transfer distance $(\mathrm{mm})$ & 10 \\
Maximum transfer distance $(\mathrm{mm})$ & 100 \\
Maximum length/diameter $(\mathrm{mm})$ & 100 \\
Maximum width $(\mathrm{mm})$ & 100 \\
Maximum whole coils area $\left(\mathrm{m}^{2}\right)$ & 0.01 \\
Material of coils conductor & Copper \\
\hline
\end{tabular}

Hence, the outer diameter must be at maximum according to the constraint, which is $100 \mathrm{~mm}$. Next, the value of pitch must be as small as possible, which is $0.1 \mathrm{~mm}$. The number of turns is set to 20 turns as the further increase will make the outer diameter exceed the constrain. The standard width of copper wire value is $1 \mathrm{~mm}$. Then, the optimum inner diameter value can be calculated on Eq. 2.

$$
D_{i}=D_{o}-2 N(w+p)
$$

The last optimization process that can be made is the implementation of ferrite shielding. Ferrite flat plate and aluminum sheet are suitable for CC-coil shape. The inner radius of the ferrite plate is $50 \mathrm{~mm}$ with a height of $2 \mathrm{~mm}$. An aluminum sheet is placed after the ferrite plate with a size $120 \mathrm{~mm} \times 165 \mathrm{~mm} \times 2 \mathrm{~mm}$. The transmitter and receiver coils must exceed the 0.3 coupling coefficient to determine the optimal distance of the circuit on vertical and horizontal misalignment. The representation of vertical and horizontal misalignment is shown in Fig. 3. 

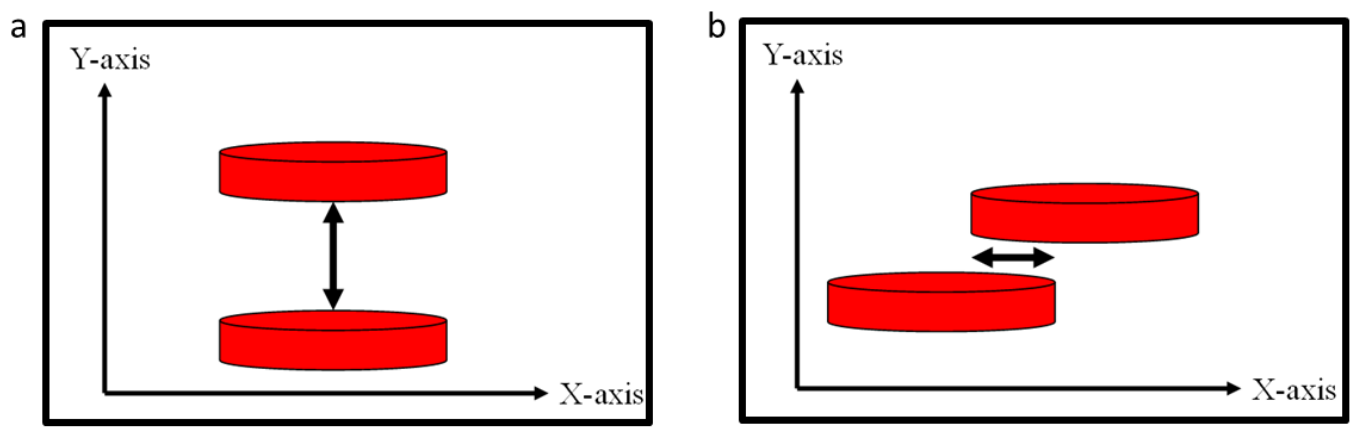

Fig. 3 - (a) Vertical misalignment; (b) Horizontal misalignment

\subsection{Power Electronics Circuit}

Power electronics circuit is essential to generate the high frequency up to $\mathrm{MHz}$ that will be resonant frequency. The power electronics circuit used in this study is a full-wave AC-DC converter (rectifier) and DC-AC converter (inverter). There are two rectifiers in the WPT system, which are at transmitter part and receiver part.

\section{(a) Full-wave AC-DC converter}

Full-wave AC-DC converter or full-wave rectifier converts AC source from the grid (230 Vrms, $50 \mathrm{~Hz})$ to DC. Since the DC source from the rectifier contains a high ripple percentage, LC-filter is crucial to reduce it. The formulation of the LC filter is shown in Eq. 3. The value of the inductor in the filter is determined using try-and-error based on [13] to reduce the loading effect of the circuit. The circuit is drawn and assemble with Ansys Twin Builder 2019 R3 simulator under the Simplorer component.

$$
\text { Ripple Factor }=\frac{0.707}{2 f_{r} R C-1}
$$

\section{(b) Full-wave DC-AC converter}

After the AC source is rectified by rectifier, DC-AC converter or inverter is used to convert back to AC source but in resonant frequency. The DC-AC converter is controlled by using the PWM block in Ansys Twin Builder 2019 R3. The simulation setting is still the same as the AC-DC converter. However, generation for the 3-level inverter is needed to decrease total harmonic distortion (THD). The 3-level inverter can be done by altering the duty cycle and phase-shift the PWM. Fig. 4 shows the generation of a 3-level inverter [14].

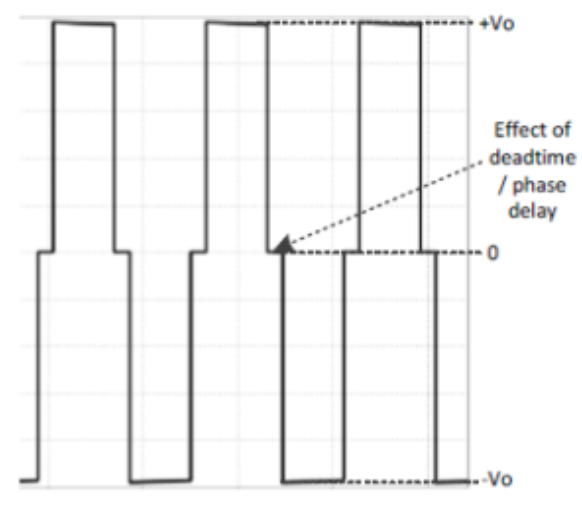

Fig. 4 - (a) Vertical misalignment; (b) Horizontal misalignment

\subsection{Series-Series (S-S) Magnetic Resonance Compensation Circuit}

The determination of high-frequency tuned during resonant is crucial as it will affect the WPT performance. ISM has allocated the frequency band for the WPT circuit, which is $6.78 \mathrm{MHz}, 13.56 \mathrm{MHz}, 27.284 \mathrm{MHz}$, and $40.7 \mathrm{MHz}$. For this study, the $6.78 \mathrm{MHz}$ frequency band is chosen as the efficiency of the WPT is better than other frequency bands. Fig. 5 shows the S-S magnetic resonance compensation circuit. The value of compensation value at a resonant 
frequency can be obtained in Eq. 4. $\omega_{\mathrm{n}}$ is represented as the natural angular resonant frequency. Moreover, $\mathrm{L}_{1}$ and $\mathrm{L}_{2}$ represented the value of the inductor at transmitter and receiver that shown in Fig. 5.

$$
\omega_{n}=\frac{1}{\sqrt{L_{1} C_{1}}}=\frac{1}{\sqrt{L_{2} C_{2}}}
$$

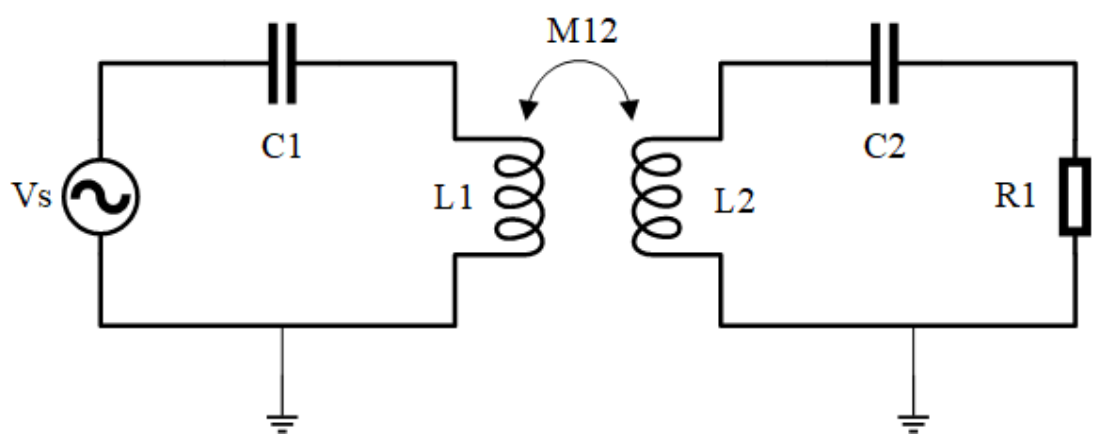

Fig. 5 - S-S compensation circuit

CC-coils from the Ansys Electronics Desktop under Maxwell 3D component can be co-simulated with the Ansys Twin Builder under Simplorer by transforming the model into the block diagram. Simplorer used a transient analysis (TR) setup with the end time $\left(\mathrm{T}_{\text {end }}\right)$ of $10 \mathrm{~ms}$, minimum time step $\left(\mathrm{H}_{\min }\right)$ of $100 \mathrm{ps}$ with maximum time step $\left(\mathrm{H}_{\max }\right)$ of 1 us. The circuit's input impedance can be obtained using Kirchoff Voltage Law (KVL) shown in Eq. 5 [15].

$$
Z_{\text {in }}=\frac{\left[r_{1}+j\left(\omega L_{1}-\frac{1}{\omega C_{1}}\right)\right]\left[r_{2}+R_{L}+j\left(\omega L_{2}-\frac{1}{\omega C_{2}}-\frac{1}{\omega C_{f}}\right)\right]+\omega^{2} L_{m}^{2}}{r_{2}+R_{L}+j\left(\omega L_{2}-\frac{1}{\omega C_{2}}-\frac{1}{\omega C_{f}}\right)}
$$

\section{Results and Analysis}

The design of CC-coil with shielding and without shielding is simulate using Ansys Electronics Desktop 2019 R3 (Maxwell 3D) for coupling coefficient comparison. The optimal distance of the CC-coil is determined by vertical and horizontal misalignment. Then, the WPT system circuit with CC-coil is simulated using Ansys Twin Builder 2019 R3 (Simplorer) to observe the power transfer efficiency (PTE).

\subsection{Simulation of CC-coil}

Based on the previous section, the CC-coil is optimized for geometry and ferrite with aluminum shielding. The optimized parameter in terms of the geometry of CC-coil is shown in Table 2. Fig. 6 shows the CC-coil without shielding and with shielding for comparison between the coupling coefficient.

Table 2 - Parameter of CC-coil

\begin{tabular}{cc}
\hline Parameter & Value \\
\hline Shape & Circular \\
Outer diameter, $\mathrm{d}_{\text {out }}$ & $100 \mathrm{~mm}$ \\
Inner diameter, $\mathrm{d}_{\text {in }}$ & $56.2 \mathrm{~mm}$ \\
Pitch, $\mathrm{s}$ & $0.1 \mathrm{~mm}$ \\
Width of wire, $\mathrm{w}$ & $1 \mathrm{~mm}$ \\
Number of turns, $\mathrm{N}$ & 20 \\
Ferrite radius & $50 \mathrm{~mm}$ \\
Aluminum sheet size & $120 \mathrm{~mm} \times 165 \mathrm{~mm} \times 2 \mathrm{~mm}$ \\
\hline
\end{tabular}



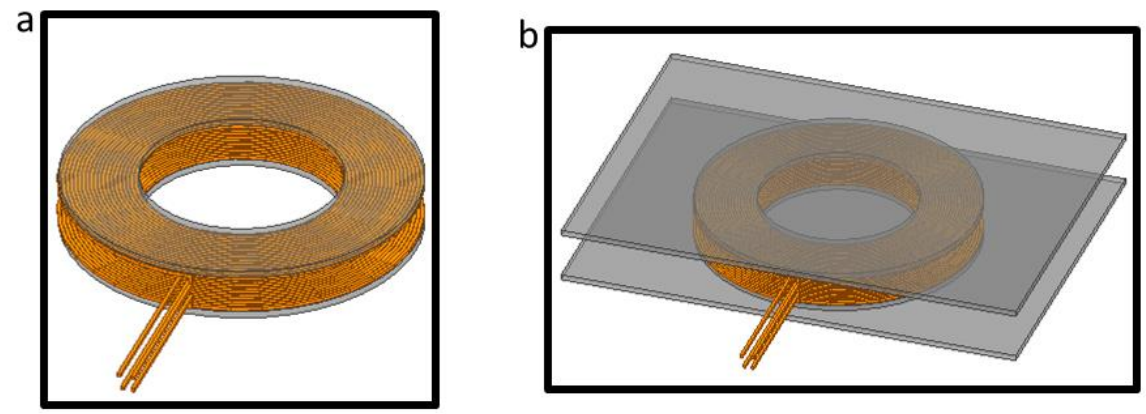

Fig. 6 - Transmitter and receiver of CC-coil (a) without shielding (b) with shielding

The simulation shows that the coupling coefficient of the CC-coil without shielding is 0.5811229 while CC-coil with shielding is 0.705373 . Therefore, the coupling coefficient of the CC-coil increases by $21.38 \%$. The magnetic field contour in Fig. 7 shows that the magnetic field for unshielded coil scatters compared to the shielded coil that focuses between transmitter and receiver. Shielding also reduced the effect of electromagnetic interference (EMI) from the surrounding that affected the coupling coefficient of the coil. Therefore, it is essential to consider shielding when designing the transmitter and receiver coil as it can hugely improve the coupling coefficient between transmitter and receiver.
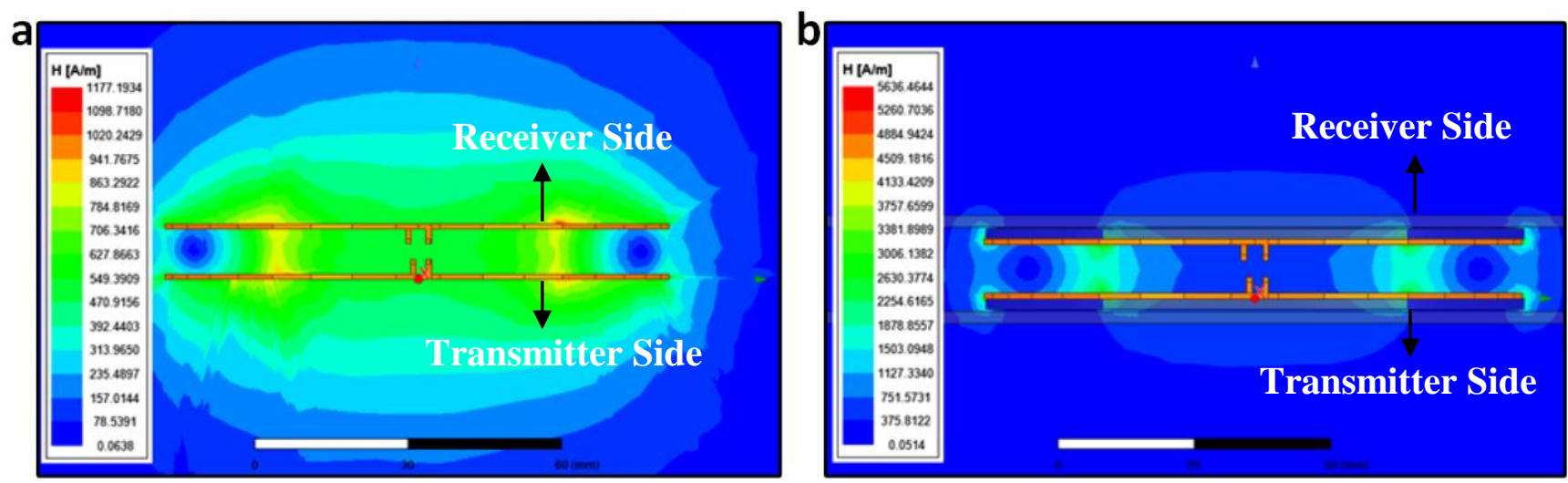

Fig. 7 - Contour of magnetic field for (a) unshielded coil; (b) shielded coil

Fig. 8 show the horizontal and vertical misalignment of $\mathrm{CC}$-coil to determine the optimal distance. The optimal distance also can be said a high-efficiency distance (HED). The HED for CC-coil for vertical misalignment from 0 mm to $30 \mathrm{~mm}$. HED for horizontal misalignment from $0 \mathrm{~mm}$ to $37 \mathrm{~mm}$. There is a null point condition in horizontal misalignment in which the coupling coefficient becomes negative that starts from $70 \mathrm{~mm}$. The flux will leave the surface of one sub-coil and reach the surface of the other when the continuous current supplies the CC-coil. The righthand thumb rule can illustrate this. The value of self and mutual inductance varies with transfer distance in misalignment analysis, but the variation rate is much lower than the supply current [16]. Therefore, the magnetic flux density generated by the coil and the flux can be modelled as signed peak amplitude (SPA) shown in Fig. 9.

For the aligned coil, the SPA can be denoted as positive. However, as the receiver coil moves to the right in horizontal misalignment, the coil's flux is weakened. Then, the SPA of the coil will drop to zero and become negative. Theoretically, as the coil moves further to the right, the SPA of the coil will become zero back as there is no electromagnetic induction between the transmitter and receiver. The transfer distance observation of the horizontal misalignment has been increased up to $110 \mathrm{~mm}$ in this study. It can be seen the pattern of coupling coefficient back to zero coincided with SPA theory. Null-point also is impossible to happen in vertical misalignment as transmitter and receiver position are always aligned (positive SPA) except their transfer distance varied vertically. 


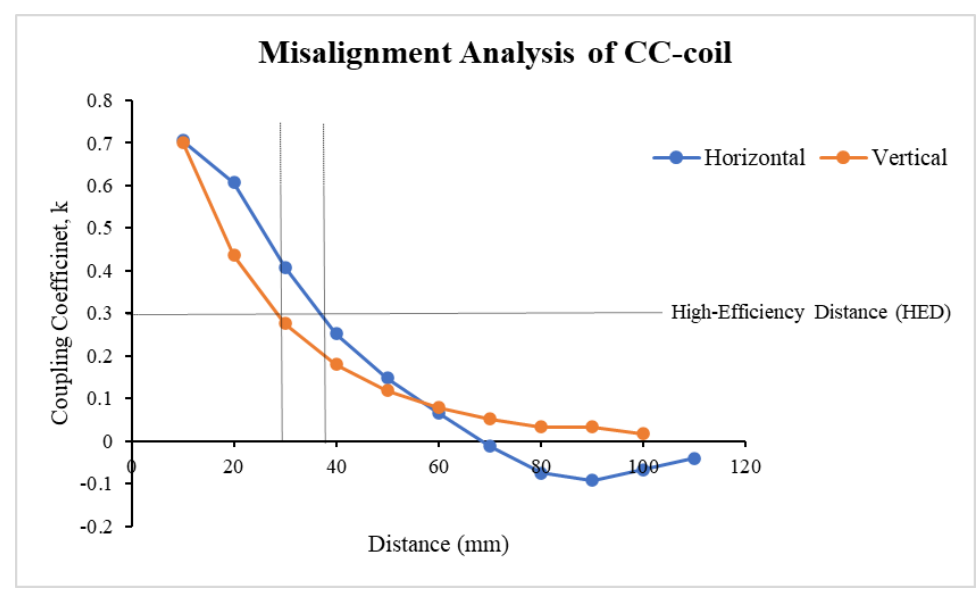

Fig. 8 - Horizontal and vertical misalignment analysis of CC-coil

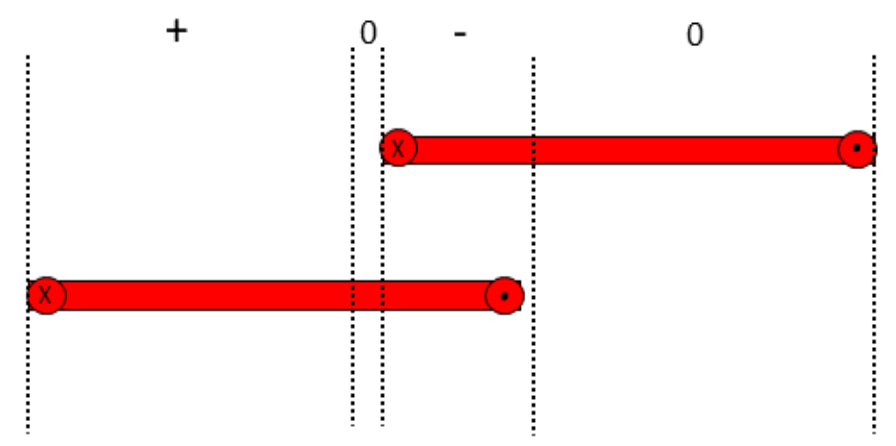

Fig. 9 - Horizontal misalignment of CC-coil with SPA signed

\subsection{Simulation of the WPT System}

WPT system circuit comprises all components discussed in the previous section. The transfer distance for the WPT system will be considered from $0 \mathrm{~mm}$ to $50 \mathrm{~mm}$ for vertical misalignment only to make analysis easier. Therefore, the compensation circuit's optimal value needs to be determined since the value of self and mutual inductance varied with the transfer distance. Based on Eq. 4, each value of the compensation circuit is calculated and observed in terms of power transfer efficiency (PTE) and power received. The most feasible value of the compensation circuit is at $50 \mathrm{~mm}$ as it can maintain good PTE and power received. Table 3 shows the critical parameter in the WPT system circuit. Fig. 10 shows the WPT system circuit in Simplorer.

Table 3 - Parameter for WPT system circuit

\begin{tabular}{cc}
\hline Parameter & Value \\
\hline Voltage input, $V_{\text {in }}$ & $230 \mathrm{~V}_{\mathrm{rms}}$ \\
Supply frequency, $f_{\text {in }}$ & $50 \mathrm{~Hz}$ \\
Inverter switching frequency, $f_{s w}$ & $6.78 \mathrm{MHz}$ \\
Inverter PWM duty cycle, $D$ & 0.49 \\
Inverter phase shift & $-18^{\circ}$ \\
Resonant frequency, $f_{r}$ & $6.78 \mathrm{MHz}$ \\
Transmitter compensation, $C_{l}$ & $8.5779 \mathrm{pF}$ \\
Receiver compensation, $C_{2}$ & $8.5498 \mathrm{pF}$ \\
Transmitter side LC filter, $L_{f_{-} T x} \& C_{f_{-} T x}$ & $125 \mathrm{uH}, 3 \mathrm{Mf}$ \\
Receiver side LC filter, $L_{f_{-} R x} \& C_{f_{-} R x}$ & $1 \mathrm{nH}, 2 \mathrm{uF}$ \\
Load Resistor, $R_{L}$ & $150 \Omega$ \\
\hline
\end{tabular}




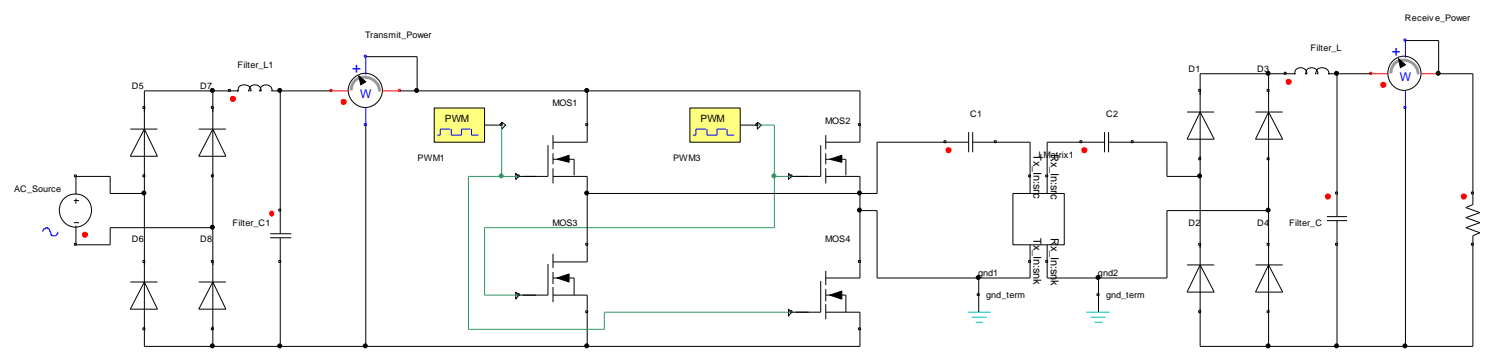

Fig. 10 - WPT system circuit

Table 4 shows the simulation result for the CC-coil using S-S magnetic resonance compensation technique. The result indicates that the output power and power transfer efficiency (PTE) increase as the transfer distance increases. Theoretically, the output power and PTE will increase to a certain and decrease back. However, the output power and PTE are not reduced when transfer distance is up to $50 \mathrm{~mm}$ shows that this topology is good with misalignment. Figure 11 and 12 shows the output waveform of voltage with current at $10 \mathrm{~mm}$ and $50 \mathrm{~mm}$ transfer distance. From Fig. 11 and 12 , the output voltage and current ripple is less than $0.5 \%$ from the $10 \mathrm{~mm}$ transfer distance until $50 \mathrm{~mm}$. The output waveform of the $10 \mathrm{~mm}$ has estimated step-response for $3.2 \mathrm{~ms}$ before steady-state, while the step-response for $50 \mathrm{~mm}$ is $3.8 \mathrm{~ms}$. The step-response of the WPT system is influenced by the input impedance and the size of the LC filter. Generally, if the LC filter is designed for a specific transfer distance, the step response can be reduced by less than 1 $\mathrm{ms}$, but it is not feasible for other transfer distances. Implementation of control circuits in the rectifier and inverter can also improve the system's step-response.

Table 4 - Simulation of WPT system for CC-coil with S-S compensation

\begin{tabular}{cccccccc}
\hline $\begin{array}{l}\text { Transfer } \\
\text { Distance } \\
(\mathbf{m m})\end{array}$ & $\begin{array}{l}\text { Input } \\
\text { voltage } \\
(\mathbf{V})\end{array}$ & $\begin{array}{l}\text { Input } \\
\text { current } \\
(\mathbf{A})\end{array}$ & $\begin{array}{l}\text { Output } \\
\text { voltage } \\
(\mathbf{V})\end{array}$ & $\begin{array}{l}\text { Output } \\
\text { current }(\mathbf{A})\end{array}$ & $\begin{array}{l}\text { Input } \\
\text { power } \\
(\mathbf{W})\end{array}$ & $\begin{array}{l}\text { Output } \\
\text { power } \\
(\mathbf{W})\end{array}$ & $\begin{array}{l}\text { PTE } \\
(\%)\end{array}$ \\
\hline 10 & 230 & 0.0902 & 39.9759 & 0.2665 & 19.1761 & 10.6538 & 55.5577 \\
20 & 230 & 0.05176 & 35.2450 & 0.2350 & 13.1504 & 8.2814 & 62.9748 \\
30 & 230 & 0.0802 & 48.229 & 0.32151 & 22.1337 & 15.5056 & 70.0542 \\
40 & 230 & 0.16967 & 72.1797 & 0.4812 & 48.6653 & 34.7327 & 71.3705 \\
50 & 230 & 0.34231 & 103.979 & 0.6932 & 98.0806 & 72.0779 & 73.4485 \\
\hline
\end{tabular}
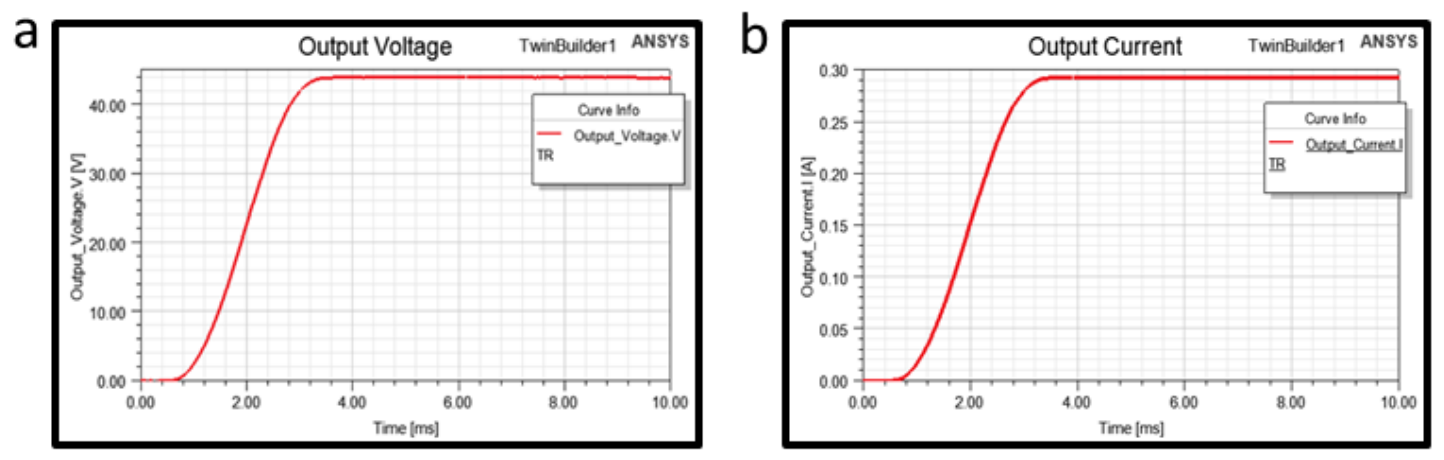

Fig. 11 - Output waveform of WPT system at $10 \mathrm{~mm}$ transfer distance (a) voltage; (b) current 

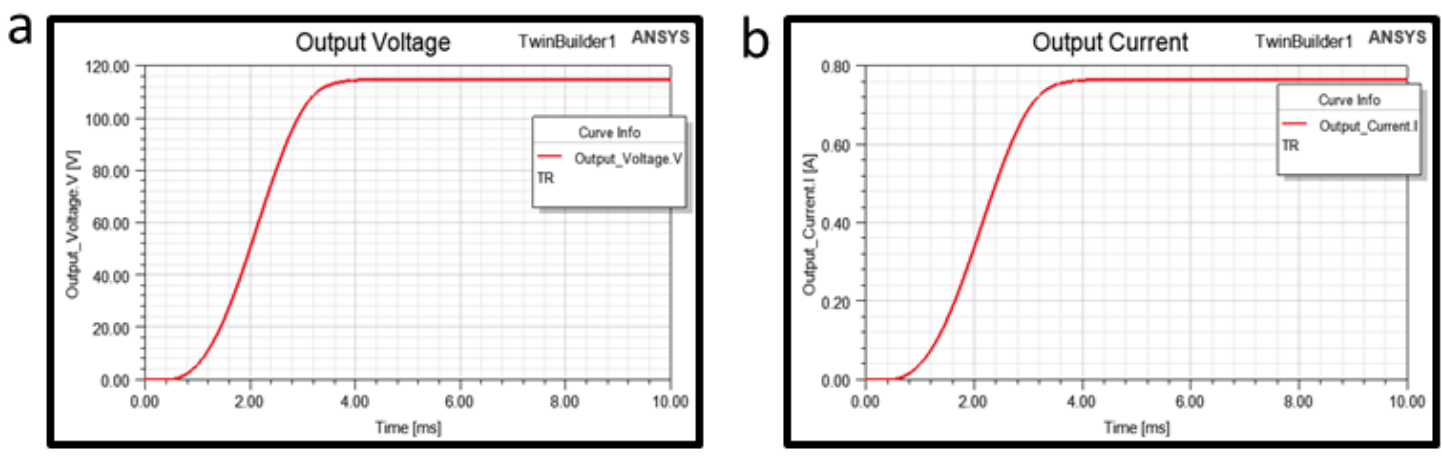

\section{Fig. 12 - Output waveform of WPT system at $50 \mathrm{~mm}$ transfer distance (a) voltage; (b) current}

The power received by the WPT circuit is more than $8 \mathrm{~W}$ shows that this circuit is only suitable for low-power applications such as the mobile phone charger. In contrast, the system input power increases as the transfer distance between the coils increases. The possible reason behind this condition is the value of inductance (self-inductance and mutual inductance) with resistance vary as the transfer distance increase. Based on Eq. 5, the input impedance of the system at $0 \mathrm{~mm}$ to $50 \mathrm{~mm}$ transfer distance can be analyzed. From Fig. 10 and 11, the output voltage and current ripple is less than $0.5 \%$ from the $10 \mathrm{~mm}$ transfer distance until $50 \mathrm{~mm}$.

Table 5 - Input impedance of CC-coil

\begin{tabular}{cc}
\hline Transfer Distance $(\mathbf{m m})$ & Absolute Input Impedance $(\boldsymbol{\Omega})$ \\
\hline 10 & $9363.55 \angle-64.394^{\circ}$ \\
20 & $6786.72 \angle-31.192^{\circ}$ \\
30 & $3003.04 \angle-9.021^{\circ}$ \\
40 & $1412.68 \angle-1.501^{\circ}$ \\
50 & $677.301 \angle-0.453^{\circ}$ \\
\hline
\end{tabular}

Since the compensation value of the circuit is set at $50 \mathrm{~mm}$, it can be seen that the angle of the impedance of CCcoil at $50 \mathrm{~mm}$ is close to zero, which represents that the circuit is purely resistive. The impedance of CC-coil from 10 $\mathrm{mm}$ to $50 \mathrm{~mm}$ is considered to be capacitive. The capacitive load caused the leading power factor on the input side. This condition can explain why the $10 \mathrm{~mm}$ compensation value of the circuit is not feasible because it will make the power factor leading that has a severe effect on the PTE. At all distances in the table, the input impedance ranges from quadrants four and one, showing expected power flows. If the transfer distance increases, the system's input impedance will go to quadrants two and three, which means reverse power transfer between transmitter and receiver.

\section{Conclusion}

This paper presents the simulation of the optimized CC-coil using Maxwell 3D and WPT system with S-S compensation using Simplorer. From the result, the optimized CC-coil with shielding has successfully increased the coupling coefficient by $21.38 \%$. Moreover, the coil's optimal distance or high-efficiency distance (HED) is determined with a maximum transfer distance of $37 \mathrm{~mm}$ on horizontal misalignment and $30 \mathrm{~mm}$ on vertical misalignment. The power transfer efficiency (PTE) of the WPT system is increased and more robust when implementing the series-series (S-S) compensation circuit. The PTE of the WPT system with output power increase as transfer distance increase. However, the input power of the system varied as the transfer distance increase. Therefore, the impedance matching technique on the WPT system circuit is for the future improvement of this study. The coils design with the compensation circuit must be considered in the WPT system design to achieve preferable and high PTE and output power.

\section{Acknowledgment}

The authors express their sincere gratitude to the Faculty of Electrical and Electronics Engineering, Universiti Tun Hussein Onn Malaysia (UTHM), for providing a conducive research platform. 


\section{References}

[1] H. Ramachandran, "Modelling of System Parameters in Wireless Power Transfer Systems," Int. J. Inf. Syst. Eng., vol. 1, no. 1, pp. 22-29, 2013, doi: 10.24924/ijise/2013.04/v1.iss1/22.29

[2] A. A. Atayero, O. Ajijola, S. I. Popoola, and V. O. Matthews, "Development of a wireless power transfer system using resonant inductive coupling," Lect. Notes Eng. Comput. Sci., vol. 2225, pp. 13-18, 2016

[3] P. S. Patil and S. K. Padaganur, "Challenging issues in wire less power transmission methods a survey," Int. J. Sci. Technol. Res., vol. 7, no. 8, pp. 136-139, 2018

[4] D. H. Tran, V. B. Vu, and W. Choi, "Design of a High-Efficiency Wireless Power Transfer System with Intermediate Coils for the On-Board Chargers of Electric Vehicles," IEEE Trans. Power Electron., vol. 33, no. 1, pp. 175-187, 2018, doi: 10.1109/TPEL.2017.2662067

[5] Y. Wang, J. Song, L. Lin, X. Wu, and W. Zhang, "Research on magnetic coupling resonance wireless power transfer system with variable coil structure," 2017 IEEE PELS Work. Emerg. Technol. Wirel. Power Transf. WoW 2017, pp. 4-9, 2017, doi: 10.1109/WoW.2017.7959403

[6] H. Wang, H. Qu, Q. Li, W. Wang, B. Tao, and T. Meng, "Design of dual-purpose non-overlapping coil sets applied to resonant magnetic coupling wireless charging system," ACM Int. Conf. Proceeding Ser., pp. 243 250, 2019, doi: 10.1145/3349341.3349410

[7] S. Chatterjee, A. Iyer, C. Bharatiraja, I. Vaghasia, and V. Rajesh, "Design Optimisation for an Efficient Wireless Power Transfer System for Electric Vehicles," Energy Procedia, vol. 117, pp. 1015-1023, 2017, doi: 10.1016/j.egypro.2017.05.223

[8] V. Shevchenko, O. Husev, B. Pakhaliuk, O. Karlov, and I. Kondratenko, "Coil design for wireless power transfer with series-parallel compensation," 2019 IEEE 2nd Ukr. Conf. Electr. Comput. Eng. UKRCON 2019 Proc., pp. 401-407, 2019, doi: 10.1109/UKRCON.2019.8879877

[9] D. Leskarac, C. Panchal, S. Stegen, and J. Lu, "PEV charging technologies and V2G on distributed systems and utility interfaces," Veh. Link. Electr. Veh. to Smart Grid, pp. 157-221, 2015, doi: 10.1049/PBPO079E_ch6

[10] W. Yan and J. Chen, "A general design of magnetic coupling resonant wireless power transmission circuit," IOP Conf. Ser. Earth Environ. Sci., vol. 69, no. 1, 2017, doi: 10.1088/1755-1315/69/1/012197

[11] H. Hashim and A. Ponniran, "Optimization of Wireless Power Transfer Configuration for High Efficiency Achievement," vol. 1, no. 1, pp. 83-94, 2020, doi: 10.30880/eeee.2020.01.01.010

[12] C. Yee Yong and K. Fen Chen, "Wireless Power Transfer Technology Using Resonant Technique," IOP Conf. Ser. Earth Environ. Sci., vol. 268, no. 1, 2019, doi: 10.1088/1755-1315/268/1/012102

[13] S. Pyakuryal and M. Matin, "Filter Design for AC to DC Converter," Int. Ref. J. Eng. Sci., vol. 2, no. 6, pp. 4249, 2013

[14] M. A. N. Kasiran, "A study on 4-level dc-dc boost inverter with passive component design consideration for renewable energy application," Universiti Tun Hussein Onn Malaysia, 2017

[15] T. Imura, U. Magnetic, E. Resonance, and C. Techniques, Wireless Power Transfer Using Magnetic and Electric Resonance Coupling Techniques, 1st Editio. Japan: Springer, 2020

[16] M. Bertoluzzo, G. Buja, and H. Dashora, "Avoiding Null Power Point in DD coils," 2019 IEEE PELS Work. Emerg. Technol. Wirel. Power Transf. WoW 2019, pp. 11-15, 2019, doi: 10.1109/WoW45936.2019.9030658 